\title{
SPECTRAL MULTIPLICITY OF SELFADJOINT DILATIONS
}

\author{
RICHARD C. GILBERT
}

Let $A$ be a closed symmetric operator in a Hilbert space $H$. If $A^{+}$ is a selfadjoint operator in a Hilbert space $H^{+}$such that $H \subset \mathrm{H}^{+}$ and $A \subset A^{+}$, then we shall call $A^{+}$a dilation of $A$. In the particular case that $H=H^{+}, A^{+}$is also called an extension of $A$. A dilation $A^{+}$is called minimal if $A^{+}$is not reduced by $H^{+} \ominus H$ nor by any of its nontrivial subspaces. For further information on selfadjoint extensions and dilations of symmetric operators, we refer the reader to Achieser and Glasmann [1].

Suppose that $A$ has deficiency indices $(1,1)$. It is the purpose of this article to show that if $\mu_{0}$ is a point of regular type for $A$, then there is a neighborhood of $\mu_{0}$ in which every minimal selfadjoint dilation of $A$ has spectral multiplicity not exceeding 1 . More precisely, there exists an interval $(\gamma, \delta)$ containing $\mu_{0}$ such that if $A^{+}$is a minimal selfadjoint dilation of $A$ with spectral function $E^{+}(\lambda)$, then the operator $A^{+}\left[E^{+}(\delta)-E^{+}(\gamma)\right]$ is unitarily equivalent to the multiplication operator in a space $L^{2}(\rho)$ for some nondecreasing function $\rho$ defined on the real line. This constitutes a generalization of Theorem 5.2 of McKelvey [4], which is in turn a generalization of Theorem 14 of Coddington [2].

As a preliminary we prove two lemmas.

Lemma 1. A real point $\mu_{0}$ is a point of regular type for a closed symmetric operator $A$ if and only if there is a selfadjoint extension of $A$ for which $\mu_{0}$ is a regular point (i.e., $a$ point in the resolvent set).

Proof. Suppose that $A_{0}$ is a selfadjoint extension of $A$ for which $\mu_{0}$ is a regular point. Since $\left(A_{0}-\mu_{0} E\right)^{-1}$ exists and is bounded it follows that $\left(A-\mu_{0} E\right)^{-1}$ exists and is bounded. Hence, $\mu_{0}$ is a point of regular type for $A$.

Suppose, on the other hand, that $\mu_{0}$ is a point of regular type for $A$. By Hartman [3, $\$ 3$, item (ii)], there exists a selfadjoint extension $A_{0}$ such that $\mu_{0}$ is not in the point spectrum of $A_{0}$. Therefore, $\left(A_{0}-\mu_{0} E\right)^{-1}$ exists. By McKelvey [4, Lemma 4.2], $\left(A_{0}-\mu_{0} E\right)^{-1}$ is bounded and has domain $H$. Hence, $\mu_{0}$ is in the resolvent set of $A_{0}$. The lemma is proved.

Lemma 2. Suppose that $A$ is a closed symmetric operator with defi-

Presented to the Society, January 5, 1967; received by the editors January 8, 1967. 
ciency indices $(1,1)$. Let $g\left(\lambda_{0}\right)$ be an element of norm 1 in the deficiency subspace of $A$ corresponding to the nonreal number $\bar{\lambda}_{0}$. Let $(\gamma, \delta)$ be a finite real interval in the resolvent set of a selfadjoint extension $A_{0}$ of $A$. Suppose that $g(\lambda)=g\left(\lambda_{0}\right)+\left(\lambda-\lambda_{0}\right) R_{0}(\lambda) g\left(\lambda_{0}\right)$ and that $\rho_{0}(\mu)$ $=\left(E_{0}(\mu) g\left(\lambda_{0}\right), g\left(\lambda_{0}\right)\right)$, where $R_{0}(\lambda)$ is the resolvent and $E_{0}(\mu)$ the spectral function of $A_{0}$. Let $\left\{\gamma_{n}\right\}$ be a decreasing sequence of real numbers approaching $-\infty$, and let $\left\{\delta_{n}\right\}$ be an increasing sequence of real numbers approaching $+\infty$, where $\gamma_{1}<\gamma<\delta<\delta_{1}$. Let

$$
h_{n}=\left\{1-\left[\rho_{0}\left(\delta_{n}\right)-\rho_{0}\left(\gamma_{n}\right)\right]\right\}^{-1}\left\{g\left(\lambda_{0}\right)-\left[E_{0}\left(\delta_{n}\right)-E_{0}\left(\gamma_{n}\right)\right] g\left(\lambda_{0}\right)\right\} .
$$

Then as $n$ approaches $+\infty,\left(g(\lambda), h_{n}\right)$ approaches 1 , uniformly for $\gamma<\lambda<\delta$.

Proof. We note first of all that since $A_{0}$ is unbounded, it follows that $\rho_{0}\left(\delta_{n}\right)-\rho_{0}\left(\gamma_{n}\right) \neq 1$, so that our definition of $h_{n}$ is legitimate.

A straightforward calculation with use of the operational calculus shows that if $\gamma<\lambda<\delta$,

$$
\begin{aligned}
\left(g(\lambda), g\left(\lambda_{0}\right)\right. & \left.-\left[E_{0}\left(\delta_{n}\right)-E_{0}\left(\gamma_{n}\right)\right] g\left(\lambda_{0}\right)\right)=1-\left[\rho_{0}\left(\delta_{n}\right)-\rho_{0}\left(\gamma_{n}\right)\right] \\
& +\left(\lambda-\lambda_{0}\right) \int_{-\infty}^{\gamma_{n}}(\mu-\lambda)^{-1} d\left(E_{0}(\mu) g\left(\lambda_{0}\right), g\left(\lambda_{0}\right)\right) \\
& +\left(\lambda-\lambda_{0}\right) \int_{\delta_{n}}^{\infty}(\mu-\lambda)^{-1} d\left(E_{0}(\mu) g\left(\lambda_{0}\right), g\left(\lambda_{0}\right)\right) .
\end{aligned}
$$

Hence, for each $\epsilon>0$ there exists an $N$ such that if $n \geqq N$ and $\gamma<\lambda<\delta$,

$$
\begin{aligned}
\left|\left(g(\lambda), h_{n}\right)-1\right| \leqq\left\{1-\left[\rho_{0}\left(\delta_{n}\right)-\rho_{0}\left(\gamma_{n}\right)\right]\right\}^{-1} \\
\cdot\left\{\epsilon\left(E_{0}\left(\gamma_{n}\right) g\left(\lambda_{0}\right), g\left(\lambda_{0}\right)\right)+\epsilon\left[1-\left(E_{0}\left(\delta_{n}\right) g\left(\lambda_{0}\right), g\left(\lambda_{0}\right)\right)\right]\right\}=\epsilon .
\end{aligned}
$$

This proves the lemma.

THEOREM 1. Suppose that $A$ is a closed symmetric operator with deficiency indices $(1,1)$. Let $A_{0}$ be a selfadjoint extension of $A$. Suppose that $\left[\gamma_{1}, \delta_{1}\right]$ is a real interval in the resolvent set of $A_{0}$. Then, every minimal selfadjoint dilation of $A$ has spectral multiplicity no more than 1 in $\left[\gamma_{1}, \delta_{1}\right]$.

Proof. Let $A^{+}$be any minimal selfadjoint dilation of $A$. Let $R(\lambda)$ be the generalized resolvent and $E(\lambda)$ the spectral function of $A$ defined by $A^{+}$. As is known, for all $f, h$ in $H$ and for any real numbers $\alpha, \beta, \alpha<\beta$,

$$
\begin{aligned}
\left(\left[\frac{1}{2}\{E(\beta)\right.\right. & \left.\left.+E(\beta+0)\}-\frac{1}{2}\{E(\alpha)+E(\alpha+0)\}\right] f, h\right) \\
= & (2 \pi i)^{-1} \lim _{\tau \rightarrow+0} \int_{\alpha}^{\beta}([R(\sigma+i \tau)-R(\sigma-i \tau)] f, h) d \sigma .
\end{aligned}
$$


(See Straus [5], for example.)

According to Krein (see Achieser and Glasmann [1, Appendix I, $\S 4])$, for any nonreal $\lambda$,

$$
R(\lambda)=R_{0}(\lambda)-\left[\theta(\lambda)+Q_{1}(\lambda)\right]^{-1}(\cdot, g(\bar{\lambda})) g(\lambda) .
$$

Here $R_{0}(\lambda)$ is the resolvent of $A_{0} ; g(\lambda)=g\left(\lambda_{0}\right)+\left(\lambda-\lambda_{0}\right) R_{0}(\lambda) g\left(\lambda_{0}\right)$, Where $I \lambda_{0}>0$ and $g\left(\lambda_{0}\right)$ is an element of norm 1 in the deficiency subspace of $A$ corresponding to $\bar{\lambda}_{0} ; Q_{1}(\lambda)=i I \lambda_{0}+\left(\lambda-\lambda_{0}\right)\left(g\left(\lambda_{0}\right), g(\bar{\lambda})\right)$, and $Q_{1}(\lambda)$ has the properties that $Q_{1}(\bar{\lambda})=\left[Q_{1}(\lambda)\right]-$ and that $I Q_{1}(\lambda)>0$ for $I \lambda>0 ; \theta(\lambda)$ is an analytic function in the upper and lower halfplanes with the properties that $\theta(\bar{\lambda})=[\theta(\lambda)]^{-}$and that $I \theta(\lambda) \geqq 0$ for $I \lambda>0$. All of these are proved in the section just cited from Achieser and Glasmann, except the facts that $Q_{1}(\bar{\lambda})=\left[Q_{1}(\lambda)\right]$, that $I Q_{1}(\lambda)>0$ for $I \lambda>0$, and that $\theta(\bar{\lambda})=[\theta(\lambda)]^{-}$. Here [ ]- stands for complex conjugate.

One may check that $Q_{1}(\bar{\lambda})=\left[Q_{1}(\lambda)\right]^{-}$as follows: Substituting the expression for $g(\lambda)$ in to $Q_{1}(\bar{\lambda})$, we obtain

$$
\begin{aligned}
Q_{1}(\bar{\lambda}) & =i I \lambda_{0}+\left(\bar{\lambda}-\lambda_{0}\right)\left(g\left(\lambda_{0}\right), g\left(\lambda_{0}\right)\right)+\left(\bar{\lambda}-\lambda_{0}\right)\left(\bar{\lambda}-\bar{\lambda}_{0}\right)\left(g\left(\lambda_{0}\right), R_{0}(\lambda) g\left(\lambda_{0}\right)\right) \\
& =-i I \lambda_{0}+\left(\bar{\lambda}-\bar{\lambda}_{0}\right)\left(g\left(\lambda_{0}\right), g\left(\lambda_{0}\right)\right)+\left(\bar{\lambda}-\lambda_{0}\right)\left(\bar{\lambda}-\bar{\lambda}_{0}\right)\left(R_{0}(\bar{\lambda}) g\left(\lambda_{0}\right), g\left(\lambda_{0}\right)\right) \\
& =-i I \lambda_{0}+\left(\bar{\lambda}-\bar{\lambda}_{0}\right)\left(g(\bar{\lambda}), g\left(\lambda_{0}\right)\right)=\left[Q_{1}(\lambda)\right]^{-} .
\end{aligned}
$$

One may prove that $I Q_{1}(\lambda)>0$ for $I \lambda>0$ as follows:

$$
\begin{aligned}
g(\bar{\lambda}) & =g\left(\lambda_{0}\right)+\left(\bar{\lambda}-\lambda_{0}\right) R_{0}(\bar{\lambda}) g\left(\lambda_{0}\right)=\left(A_{0}-\lambda_{0} E\right)\left(A_{0}-\bar{\lambda} E\right)^{-1} g\left(\lambda_{0}\right) \\
& =\int_{-\infty}^{\infty}\left(\mu-\lambda_{0}\right)(\mu-\bar{\lambda})^{-1} d E_{0}(\mu) g\left(\lambda_{0}\right),
\end{aligned}
$$

where $E_{0}(\mu)$ is the spectral function of $A_{0}$. Hence,

$$
\begin{aligned}
Q_{1}(\lambda) & =i I \lambda_{0}+\left(\lambda-\lambda_{0}\right) \int_{-\infty}^{\infty}\left(\mu-\bar{\lambda}_{0}\right)(\mu-\lambda)^{-1} d\left\|E_{0}(\mu) g\left(\lambda_{0}\right)\right\|^{2} \\
& =i I \lambda_{0}+\int_{-\infty}^{\infty}\left[\left(\bar{\lambda}_{0}-\mu\right)+\left|\mu-\lambda_{0}\right|^{2}(\mu-\lambda)^{-1}\right] d\left\|E_{0}(\mu) g\left(\lambda_{0}\right)\right\|^{2} .
\end{aligned}
$$

The last equation implies that if $\lambda=x+i y$, then,

$$
I Q_{1}(\lambda)=y \int_{-\infty}^{\infty}\left|\mu-\lambda_{0}\right|^{2}|\mu-\lambda|^{-2} d\left\|E_{0}(\mu) g\left(\lambda_{0}\right)\right\|^{2}>0 .
$$

That $\theta(\bar{\lambda})=[\theta(\lambda)]-$ is proved by using the expression

$$
\theta(\lambda)=-\|g(\bar{\lambda})\|^{2}\|g(\lambda)\|^{2}\left\{\left(\left[R(\lambda)-R_{0}(\lambda)\right] g(\bar{\lambda}), g(\lambda)\right)\right\}^{-1}-Q_{1}(\lambda),
$$

which follows from equation (2). 
If we substitute (2) into (1), we obtain

$\left(\left[\frac{1}{2}\{E(\beta)+E(\beta+0)\}-\frac{1}{2}\{E(\alpha)+E(\alpha+0)\}\right] f, h\right)$

$$
\begin{array}{r}
=-(2 \pi i)^{-1} \lim _{\tau \rightarrow+0} \int_{\alpha .}^{\beta}\left\{\left[\theta(\sigma+i \tau)+Q_{1}(\sigma+i \tau)\right]^{-1}(f, g(\sigma-i \tau))\right. \\
\cdot(g(\sigma+i \tau), h)-\left[\theta(\sigma-i \tau)+Q_{1}(\sigma-i \tau)\right]^{-1} \\
\cdot(f, g(\sigma+i \tau))(g(\sigma-i \tau), h)\} d \sigma .
\end{array}
$$

If we put $\Psi(\lambda)=(f, g(\bar{\lambda}))(g(\lambda), h)$ and $\Phi(\lambda)=-\left[\theta(\lambda)+Q_{1}(\lambda)\right]^{-1}$ and suppose that $[\alpha, \beta] \subset\left[\gamma_{1}, \delta_{1}\right]$, we may use the properties of $\theta(\lambda), Q_{1}(\lambda)$ and $g(\lambda)$ to check that $\Psi(\lambda)$ and $\Phi(\lambda)$ satisfy the hypotheses of Straus [5, Lemma 5]. Hence,

$$
\begin{gathered}
\left(\left[\frac{1}{2}\{E(\beta)+E(\beta+0)\}-\frac{1}{2}\{E(\alpha)+E(\alpha+0)\}\right] f, h\right) \\
=\int_{\alpha}^{\beta}(f, g(\sigma))(g(\sigma), h) d \rho(\sigma)
\end{gathered}
$$

where

$$
\rho(\sigma)=-(1 / \pi) \lim _{\tau \rightarrow+0} \int_{0}^{\sigma} I\left[\theta(\eta+i \tau)+Q_{1}(\eta+i \tau)\right]^{-1} d \eta .
$$

It follows from (4) that

$$
\left(\left[E^{+}(\beta)-E^{+}(\alpha)\right] f, h\right)=\int_{[\alpha, \beta)}(f, g(\sigma))(g(\sigma), h) d \rho(\sigma),
$$

where $E^{+}(\mu)$ is the spectral function of $A^{+}$, and the integral is in the sense of Lebesgue.

If $\Delta=[\alpha, \beta)$, we define $E^{+}(\Delta)$ by the equation $E^{+}(\Delta)=E^{+}(\beta)-E^{+}(\alpha)$. Let $\Delta_{0}=\left[\gamma_{1}, \delta_{1}\right)$. As in McKelvey [3, Lemma 3.1], the closed linear hull of $Z\left(\Delta_{0}\right)=\left\{E^{+}(\Delta) h: \Delta \subset \Delta_{0}, h \epsilon H\right\}$ is $E^{+}\left(\Delta_{0}\right) H^{+}$.

We define the operator $V$ on $Z\left(\Delta_{0}\right)$ into $L_{\rho}^{2}\left[\gamma_{1}, \delta_{1}\right)$ as follows: $V E^{+}(\Delta) h=\chi_{[\alpha, \beta)}(\lambda)(h, g(\lambda))$, where $\Delta=[\alpha, \beta) \subset\left[\gamma_{1}, \delta_{1}\right)$, and $\chi_{[\alpha, \beta)}(\lambda)$ is the characteristic function of $[\alpha, \beta)$. We then extend $V$ linearly to the linear hull of $Z\left(\Delta_{0}\right)$. Using Lemma 2 , it is not difficult to check that if $E^{+}(\Delta) h=E^{+}\left(\Delta^{\prime}\right) h^{\prime}$, then $V E^{+}(\Delta) h=V E^{+}\left(\Delta^{\prime}\right) h^{\prime}$, and similarly for elements in the linear hull of $Z\left(\Delta_{0}\right)$. Further, from (6) it follows that $V$ is an isometry. Extending $V$ continuously to $E^{+}\left(\Delta_{0}\right) H^{+}$, we obtain an isometry from $E^{+}\left(\Delta_{0}\right) H^{+}$into $L_{\rho}^{2}\left[\gamma_{1}, \delta_{1}\right)$.

$V$ is, in fact, onto $L_{\rho}^{2}\left[\gamma_{1}, \delta_{1}\right)$. For, suppose that $k(\lambda) \in L_{\rho}^{2}\left[\gamma_{1}, \delta_{1}\right)$ and that $k(\lambda)$ is perpendicular to $\chi_{[\alpha, \beta)}(\lambda)(h, g(\lambda))$ for every $[\alpha, \beta) \subset\left[\gamma_{1}, \delta_{1}\right)$ and for all $h \in H$. Therefore, $\int_{[\alpha, \beta)}\left(h_{n}, g(\lambda)\right) k(\lambda) d \rho(\lambda)=0$ for all 
$[\alpha, \beta) \subset\left[\alpha_{1}, \beta_{1}\right)$, where $\left\{h_{n}\right\}$ is the sequence of Lemma 2. Taking the limit, it follows from Lemma 2 that $\int_{[\alpha, \beta)} k(\lambda) d \rho(\lambda)=0$ for all $[\alpha, \beta)$ $C\left[\gamma_{1}, \delta_{1}\right)$. Hence, $k(\lambda)$ is perpendicular to all step functions in $L_{\rho}^{2}\left[\gamma_{1}, \delta_{1}\right)$. The density of the step functions implies that $k(\lambda)=0$ almost everywhere. Whence, $V$ is onto.

It is not difficult to check that $V$ carries the spectral function of $A^{+} E^{+}\left(\Delta_{0}\right)$ into the spectral function of the multiplication operator in $L_{\rho}^{2}\left[\gamma_{1}, \delta_{1}\right)$. It follows that the spectrum of $A^{+} E^{+}\left(\Delta_{0}\right)$ has multiplicity 0 or 1 , depending on whether $\rho$ is constant or nonconstant in $\left[\gamma_{1}, \delta_{1}\right)$. This proves the theorem.

By using Lemma 1 and Theorem 1 we obtain the following:

Theorem 2. Suppose that $A$ is a closed symmetric operator with deficiency indices $(1,1)$. Let $\mu_{0}$ be a point of regular type for $A$. Then there is a neighborhood of $\mu_{0}$ in which every minimal selfadjoint dilation of $A$ has spectral multiplicity not exceeding 1.

REMARK. One might ask whether there might always be a neighborhood of a real point $\mu_{0}$ of regular type in which the spectral multiplicity of every minimal selfadjoint dilation is zero. This is not true, because there exists a selfadjoint extension for which $\mu_{0}$ is an eigenvalue. (See Achieser and Glasmann $[1, \S 83]$.) Further, there will in general be actual dilations (rather than extensions) for which the spectral multiplicity is different from zero in every neighborhood of $\mu_{0}$. This can be seen from the fact that there are many symmetric operators with deficiency index $(1,1)$ for which every point in the complex plane is of regular type. (For example, operators defined by formally selfadjoint first order ordinary differential operators with zero boundary conditions on a finite interval, operators defined by certain Sturm-Liouville operators on a semi-axis, and operators defined by certain Jacobi matrices.) Such a symmetric operator $A$ has an infinite number of selfadjoint dilations (actual dilations). Since a selfadjoint operator cannot have spectral multiplicity zero at every point, for each such selfadjoint dilation $A^{+}$of $A$ there will be at least one point $\mu_{0}$ (which is of necessity a point of regular type for $A$ ) in every neighborhood of which the spectral multiplicity of $A^{+}$is greater than zero. Such points will not even in general be eigenvalues, because in [2] and [4] it is shown that many of these dilations will be unitarily equivalent to the multiplication operator in the Hilbert space of Lebesgue square integrable functions on the real line.

\section{REFERENCES}

1. N. I. Achieser, and I. M. Glasmann, Theorie der linearen Operatoren im HilbertRaum, Akademie-Verlag, Berlin, 1954. 
2. E. A. Coddington and R. C. Gilbert, Generalized resolvents of ordinary differential operators, Trans. Amer. Math. Soc. 93 (1959), 216-241.

3. P. Hartman, On the essential spectra of symmetric operators in Hilbert space, Amer. J. Math. 75 (1953), 229-240.

4. R. McKelvey, The spectra of minimal self-adjoint extensions of a symetric operator, Pacific J. Math. 12 (1962), 1003-1022.

5. A. V. Straus, On spectral functions of differential operators, Izv. Akad. Nauk SSSR Ser. Mat. 19 (1955), 201-220.

\section{California State College at Fullerton}

